\title{
Ein spannendes, umfassendes Potpourri
}

\author{
Zum insgesamt 17. Mal fand der von der World Federation of Societies of Anesthesiologists (WFSA) \\ organisierte World Congress of Anaesthesiologists (WCA) statt - erstmals als virtuelles Format. \\ Es wurde aber einiges geboten, um die Interaktion zwischen den über 6.000 Teilnehmer*innen aus \\ allen Teilen der Welt zu fördern.
}

2 020 sollte der World Congress of Anaesthesiologists (WCA) in Prag über die "große Bühne" gehen. Pandemiebedingt musste er aber um ein Jahr verschoben werden und fand schließlich Anfang September dieses Jahres als rein virtuelle Veranstaltung statt. Sein Motto "CONNECT, LEARN, SHARE" entspreche dabei sehr konsequent dem Hauptanliegen des Veranstalters, erzählt Univ.-Prof. Dr. Anna Spacek, Klinische Abteilung für Allgemeine Anästhesie und Intensivmedizin, Universitätsklinik für Anästhesie, Allgemeine Intensivmedizin und Schmerztherapie an der MedUni Wien/AKH Wien, eine von drei Europa-Delegierten im Council der WFSA. Das zentrale Ziel der Weltföderation der nationalen anästhesiologischen Fachgesellschaften ist es, einen universellen Zugang zu einer sicheren anästhesiologischen Versorgung für alle Menschen zu schaffen, egal, in welchem Teil der Welt sie leben und behandelt werden. Angesichts der

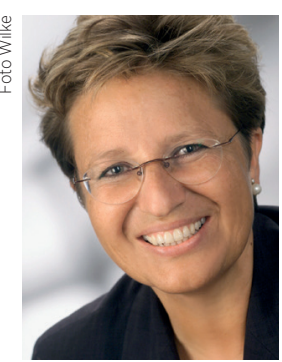

Univ.-Prof. Dr. Anna Spacek nach wie vor gro-

Ben regionalen Unterschiede im Versorgungsangebot sieht es die WFSA daher als ihre vordringliche Aufgabe, durch Spendenkampagnen, Austausch von Know-how, Fortbildungsprogramme, Programme für Gastärzt*innen und Ähnliches mehr Solidarität und Chancengleichheit für alle Patient*innen weltweit zu erreichen.

\section{ALLES FÜR ALLE}

Ein wesentliches Angebot am WCA 2021 bildeten daher die sogenannten 24 „Learning Tracks", die inhaltlich so konzipiert waren, dass sie die gesamte Themenpalette der modernen Anästhesie abdeckten: vom akuten und chronischen Schmerz, der Reanimation und Intensivmedizin, dem perioperativen Management, der Notfall- und Trau- ma-, Transplant- oder Palliativanästhesie über Spezialthemen wie Anästhesie bei Kindern, Schwangeren oder adipösen Patient*innen bis hin zu historischen und ethischen Themen. Immer im Mittelpunkt der unterschiedlichsten Sessions - als Klammer über alle Bereiche stand das Thema Patientensicherheit.

Ein wichtiger Schwerpunkt widmete sich auch der Frage der Arbeitsbedingungen für Anästhesist*innen angesichts der massiven Belastungen im Zuge der Pandemie, denn „nur wenn es den Behandler*innen gut geht, geht es auch den Patient*innen gut", so Prof. Spacek.

Alle Sessions fanden in englischer Sprache statt und stehen für registrierte Teilnehmer*innen noch bis 5. Dezember „on demand" zum Nachhören online zur Verfügung. Als besonderer Service

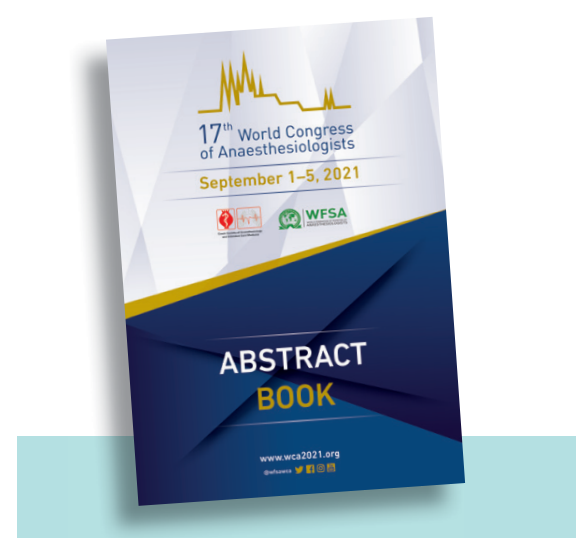

WCA 2021 IN ZAHLEN

6.539 Teilnehmer*innen aus 132 Ländern

479 Referent*innen 643 Präsentationen

1.408 Poster-Präsentationen 483 Scholars (Stipendiaten)

19 Aussteller, 14 Industrie-Sessions 24 Learning Tracks 79 Global-Village-Angebote wurden sämtliche Vorträge in Englisch, Französisch und Spanisch (jeweils zum individuellen Auswählen) untertitelt. Das habe angesichts der regionalen wie fachlichen Breite der Vortragenden und der Teilnehmer*innen laut Prof. Spacek das Erfassen der Inhalte sehr erleichtert.

\section{INTERAKTIVER AUSTAUSCH}

Trotz des Online-Formats waren die Organisator*innen sehr darum bemüht, den Teilnehmer*innen auch Plattformen anzubieten - sozusagen virtuelle Meetingräume und Kaffeehaustische -, um sich informell und auch vertraulich auszutauschen.

„Vorträge über neue Erkenntnisse, rezente Forschungsergebnisse, Leitlinienänderungen etc. sind enorm wichtig“, meint dazu Prof. Spacek. „Genauso wichtig ist es aber auch, untereinander zu kommunizieren, andere Kolleg*innen kennenzulernen und Erfahrungen aus der klinischen Praxis auszutauschen nach der Devise: Alle machen es gleich, aber doch ein bisschen anders." Außerdem würden solche Veranstaltungen eine gute Möglichkeit darstellen, um globale Allianzen zu bilden und internationale Teams für zukünftige Projekte und Studien zu formen. Die große Beteiligung der Teilnehmer*innen im Rahmen des „Global Anaesthesia Village" bestätigte schließlich die Relevanz eines solchen informellen Austausches eindrucksvoll.

Bisher wurde der WCA im 4-JahreRhythmus durchgeführt, dem WFSAAnspruch möglichst hoher Diversität und Globalität verpflichtet, breit verstreut über alle Kontinente. Derzeit wird im Council über eine Rhythmusverkürzung auf zwei Jahre beraten. Der 18. WCA wird jedenfalls 2024 in Singapur stattfinden. Ob als Präsenz- oder Hybrid-Veranstaltung, bleibt noch offen. Bericht: Mag. Volkmar Weilguni

Quelle: $17^{\text {th }}$ World Congress of Anaesthesiologists 2021, 1.-5. September 2021 (virtuell) 